\title{
Erratum: Super-resolution photoacoustic imaging through a scattering wall
}

Donald B. Conkey, Antonio M. Caravaca-Aguirre, Jake D. Dove, Hengyi Ju, Todd W. Murray \& Rafael Piestun

Nature Communications 6:7902 doi: 10.1038/ncomms8902 (2015); Published 7 Aug 2015; Updated 8 Sep 2015

The HTML version of this Article previously published omitted Supplementary Movie 1. This has now been corrected in the HTML version of the Article; the PDF was correct from the time of publication. 